\title{
Prevalence of atopic disorders in 10-15 years age group students from Kasaragod district, Kerala: A cross-sectional study
}

\author{
Madhur Guruprasad Aggithaya ${ }^{\text {a, }}$, Pradeepa P. Narayana ${ }^{b}$, K.S. Prasanna ${ }^{\text {a }}$, S.R. Narahari ${ }^{\text {a }}$ \\ ${ }^{a}$ Institute of Applied Dermatology, Uliyathadka, Madhur PO, Kasaragod, 671124, Kerala, India \\ ${ }^{\mathrm{b}}$ District TB Centre, Kasaragod, 671121, Kerala, India
}

\section{A R T I C L E I N F O}

\section{Keywords:}

Allergic rhinitis

Atopic dermatitis

Bronchial asthma

Epigenomics

International study of asthma and allergies in childhood

School health services

\begin{abstract}
A B S T R A C T
Introduction: Atopic disorders are showing a consistent trend of increasing prevalence. Kasaragod was an endosulfan aerial spray region that caused multiple health issues in the population. The aim of this paper was to a) determine the prevalence of, b) identify socio-environmental determinants (SED) of atopic disorders amongst school children of age 10-15 years in Kasaragod district.

Methodology: The study is a cross-sectional survey of students of class 8 to 10 in 20 schools of Kasaragod educational district. International Study of Asthma and Allergies in Childhood (ISAAC) core questionnaire identified atopic students. A validated, closed-ended 30 questions elicited exposure to SEDs.

Results: In 20 schools, 5914 students filled questionnaires. ISAAC questionnaire identified 2310 (41.8\%) atopic students; 716 (13\%) chronic wheezers, 543 (9.8\%) eczema and $1813(32.8 \%)$ rhinitis. The study showed a significant relationship between rhinitis mode of going to school, solid biomass fuel, rodents and reptiles infestation in living places. Rhinitis is prevalent in students who lived near the coastal region, tarred roads and footpaths. The chance of getting asthma, when a student had eczema and vice-versa (odds ratio $=2.388, \mathrm{x}^{2}=$ $66.450, \mathrm{p}=0.000$ ), between asthma to rhinitis (odds ratio $=3.671, \mathrm{x}^{2}=270.829, \mathrm{p}=0.000$ ) and eczema and rhinitis (odds ratio $=2.852, \mathrm{x}^{2}=139.485, \mathrm{p}=0.000$ ) showed significant relation.

Discussion: Atopy prevalence is higher in Kasaragod and association with other allergic disorders suggestive of atopic march. Further studies required to identify reasons; mainly on environmental pollution, epigenetic changes in students.
\end{abstract}

\section{Introduction}

Bronchial asthma is an allergic disease and is associated with other complex paradigmatic genetic disorders such as atopic dermatitis and allergic rhino-conjunctivitis. Atopic dermatitis has a predilection for infants and young children while asthma dominates in older schoolgoing children and allergic rhinitis predominates in adolescents. Atopic dermatitis affects $3 \%-20.5 \%$ of the population, and allergic rhinitis affects between $10 \%$ and $30 \% .{ }^{1}$ There is a region-wise difference in the prevalence of asthma. $4-19 \%$ is the range of prevalence of asthma in India in the age group of 10-15 years in different studies. ${ }^{2}$ The prevalence rate of asthma in South India is $10.3 \%,{ }^{3}$ Rajasthan is $7.6 \%,{ }^{4}$ and in Karnataka, it was $8.5 \% .^{5}$

This paper estimates the prevalence of atopic disorders among school children of age 10-15 years in Kasaragod educational district, and its socio-environmental determinants (SED).

\section{Methodology}

The cross-sectional study was done in the Kasaragod educational sub-district comprising the geographical areas of Kasaragod taluk from $26^{\text {th }}$ May 2016 to $30^{\text {th }}$ May 2018. The study used the International Study of Asthma and Allergies in Childhood (ISAAC) core questionnaire to identify asthma, eczema and rhinitis. ${ }^{6}$ We used the regional language (Kannada and Malayalam) translation version. It was validated and used in a single school study and published previously. ${ }^{5}$ Another questionnaire on SEDs was administered along with ISSAC to all students (Box 1). The closed-ended SED questionnaire listed known SEDs from atopic papers through a PubMed search. An expert committee, comprising of two respiratory medicine specialists, a public health expert, one district education officer and heads of two schools examined the questionnaire and approved its use. A pilot test of the SED questionnaire with 30 questions was performed on ten students of 10-15 years of age group

\footnotetext{
* Corresponding author.

E-mail address: iadorg@gmail.com (M.G. Aggithaya).
} 


\title{
Box 1
}

The socio environmental determinants for atopic disorders listed through PubMed search*

\begin{abstract}
Pollens of Amaranthus Spinosus (spiny amaranth), Parthenium hysterophorus (Parthenium Weed), Borassus flabellifer (toddy palm), Delonix regia (Gulmohar tree), Peltophorum pterocarpum (Copperpod tree), Helianthus annuus L (Sunflower), exposure to pets and cockroaches, parental smoking, traffic, Kerosene, Liquid petroleum gas and biomass fuel, urinary bisphenol, arsenic exposure, dust aerosol, aspergillus, house-dust mite, polycyclic aromatic hydrocarbons, poor housing conditions and overcrowding, dust wheat and rice, dander, cotton mill dust, saw dust (wood dust) and different types of fabrics

*PubMed search strategy used as on June 2016; '(Asthma OR Bronchial asthma OR Intrinsic asthma OR Extrinsic asthma) OR (Eczema OR Atopic dermatitis OR Atopic eczema OR Besnier's disease OR Besnier's prurigo) OR (rhinitis OR Allergic rhinitis OR common cold OR coryza OR rhinitis OR Hay fever OR rhinorrhea) AND Environment* AND India'
\end{abstract}

and modified to make questions simple self-administered for students and their patents to understand it.

The students of 5 th to 10 th standard included in the study. An education session on information about the study and health education regarding the atopic disorders were given at each class. The information provided to students and teachers in the class included symptoms, management and prevention of atopic disorders. The study team recorded height and weight and peak expiratory flow (PEF) using a peak flow meter. PEF recorded using a disposable mouthpiece for the best of three readings. Each session needed one to $2 \mathrm{~h}$ to evaluate the students from one class. We screened all willing students in the class during the day of the visit. Students received information sheet, brochures in local languages along with ISAAC \& SED questionnaire. They carried them home and filled the questionnaire with the assistance of parents and returned to the designated class teacher the next morning. We telephoned parents to fill the missing data.

The ISAAC questionnaire recommends standard methods to screen atopic disorders. Chronic wheezers were those who answered 'yes' to the following questions: 'Have you ever had wheezing or whistling in the chest at any time in the past?' and 'Have you had wheezing or whistling in the chest in the past 12 months?'. The students with allergic rhinitis were those who answered 'yes' to the following questions: 'Have you ever had a problem with sneezing or a runny, or blocked nose when you DID NOT have a cold or the flu?', 'In the past 12 months, have you had a problem with sneezing, or a runny, or blocked nose when you DID NOT have a cold or the flu?' and 'In the past 12 months, has this nose problem been accompanied by itchy-watery eyes?'. The students affirmative to eczema were those answered yes to question 'Have you ever had an itchy rash which was coming and going for at least six months?', 'Have you had this itchy rash at any time in the past 12 months?'. The students who had any, two or all three diseases of atopic disorders were considered as atopic.

The normal Indian range of PEF rate (PEFR) was obtained through 'breathe free', an initiative of the Cipla Pharmaceuticals, New Delhi.

\subsection{Statistical methods}

Out of 176 schools in the Kasaragod educational sub-district, we selected 86 schools which have the students of 10-15 years. The reasons for exclusion are; 69 lower primary schools were excluded from our target group since students were below ten years. Out of 107 schools, one is blind school, the other one is the school for deaf and dumb, and the third one is the special school for mentally disabled students. Studies with students with special abilities required separate ethical approval. There were three girl's high schools, and 14 schools had less than three classes, either up to fifth standard or up to eighth standard. These schools were excluded from the study to avoid misappropriation in gender as well as age ratio. Out of 86 selected schools, 36 were government, 17 aided and 33 unaided schools, as stratified by the educational department. The sample included one school (Sri Gopalakrishna
High School, Kudlu) from endosulfan aerial spay free Madhur panchayath. Since the sample size is large, we followed the cluster sampling method for selecting the clusters from these strata. The total number of students in the selected 86 schools is 31,447 , i.e. 13,362 (42.5\%) students in government schools, 10,541 (33.5\%) in aided and 7544 (24\%) in unaided schools. Considering the previous studies, ${ }^{2}$ we postulated the prevalence as $10 \%$ for our study to have power at $90 \%$ and 1.96 critical value at $95 \%$ confidence level. Considering one and a half design effect (1.5 times) and a 10\% drop out value, the final sample size was calculated as 5800 . Depending on the percentage of each stratum, 8, 7 and 5 schools, with an estimation of 300 students from a school, were selected in Government, aided and unaided schools respectively. Descriptive statistics used to find out the prevalence of atopic disorders among students. Chi-square test and ANOVA used to identify the SEDs. SPSS Version 16 (SPSS, Inc., Chicago, IL, USA) performed logistic regression and data analysis.

Ethical clearance obtained ${ }^{8}$ and informed consent was taken by the students before screening with approval signature from parents who crosschecked the answers given by students. We also obtained permission from officials of the education department and respective schools for screening the students.

\section{Results}

Twenty-two screening camps were conducted in 20 schools. Out of 7660 questionnaires distributed, we received 5914 (77.2\%) completed questionnaires. The response from the students was Government (77.5\%), Government aided schools $(78.7 \%)$ and unaided schools (89.1\%). Out of 5914 students screened through ISSAC, 2310 (41.8\%) showed positive symptoms of atopic disorders. Among them 716 (13\%) were chronic wheezers, 543 (9.8\%) had eczema, 1813 (32.8\%) suffered from rhinitis (Fig. 1).

\subsection{Association of social and environmental determinants (SEDs)}

SEDs listed in our questionnaire showed significant relation with eczematous ( $543=9.8 \% ; X^{2}$ value $\left.=6.844, \mathrm{P}=0.005\right)$ students and transport used to go to school (Table 1) and rhinitis (1813 (32.8\%; $\mathrm{X}^{2}$ value $=10.721, \mathrm{p}=0.001)$ to category of school they are studying $\left(\mathrm{X}^{2}\right.$ value $=33.391, \mathrm{p}=0.000$ ). Additionally, the significant association found between rhinitis and exposure to vehicle/generator smoke at home, mode of going to school, solid biomass fuels, rodents and reptiles in surroundings. Location of the house also had an influence since there is a significant relation between rhinitis and living place near the coastal region, tarred roads and footpath. Table 2 shows the logistic regression analysis of these factors with rhinitis. However, none in the SED list had a significant relation in bronchial asthma students $(716=13 \%)$.

Bronchial asthma (140 students $=13.8 \% ; \mathrm{X}^{2}$ value $=0.572, \mathrm{p}=$ 0.240 ) and eczema ( $98=9.6 \% ; X^{2}$ value $\left.=4.437, p=0.109\right)$ students missed school days giving health reasons but didn't relate statistically. 
But, $376(37 \%)$ students missed school days due to rhinitis $\left(\mathrm{X}^{2}\right.$ value $=$ 10.721, $\mathrm{p}=0.001$ ). Father's occupation and rhinitis showed statistical significance $\left(\mathrm{X}^{2}\right.$ value $\left.=43.331, \mathrm{p}=0.000\right)$, which may imply the impact of low economic status on rhinitis.

Out of 716 asthmatic students, 375 (52.5\%) students recorded low PEF Rate. There was no relation between PEFR of asthmatic students with height $(\mathrm{F}=1.440, \mathrm{p}=0.144)$, but significant at $5 \%$ level for bodyweight $(\mathrm{p}=.020)$.

The analysis revealed a very significant relationship between bronchial asthma, eczema and rhinitis. Out of 716 asthmatic students, 125 (17.5\%) had rhinitis, $62(8.7 \%)$ had eczema prior to asthma. In 543 (9.8\%) eczematous students, 75 (13.9\%) had the previous history of asthma, and 101 (18.6\%) had rhinitis. Out of 1813 (32.8\%) rhinitis students, $192(10.6 \%)$ gave a history of asthma, and $89(4.9 \%)$ had eczema before rhinitis. This finding shows the possibility of the atopic march, meaning a high possibility of getting other forms of atopic diseases when a student suffered from one disease. The chance of getting asthma, when a student has eczema and vice-versa (odds ratio $=2.388$, $\mathrm{x}^{2}=66.450, \mathrm{p}=0.000$ ), between asthma to rhinitis and vice-versa (odds ratio $\left.=3.671, \mathrm{x}^{2}=270.829, \mathrm{p}=0.000\right)$ and eczema and rhinitis and vice-versa (odds ratio $=2.852, \mathrm{x}^{2}=139.485, \mathrm{p}=0.000$ ) shows very significant relation.

The demographic details of the 5914 students are illustrated in Table 3. It shows that 5587 (94.5\%) students belonged to the economic class of annual income below INR 150000. Gender, age, economic status (category of school), had no association in our atopic students. Table 4 lists the reported food allergy and its symptoms. 1006 (17.01\%) students observed allergic reactions. There is significant association with food allergy and eczema (odds ratio $=1.457, x^{2}=7.933, p=0.002$ ). 263 (26.1\%) students specified the food items which they and their parents believed to cause food allergy. 94 (18.2\%) students did not mention the specific symptoms or food items of allergy, although parents believed that student additionally suffered from food allergy. There were no significant differences between the SED of schools from endosulfan affected area and others.

\section{Discussion}

Among the 5914 students of age group 10-15 years in the Kasaragod educational district, ISAAC questionnaire analysis showed 2310 (41.8\%) suffering from atopic disorders; 716 (13\%) chronic wheezers, 543 (9.8\%) eczema and $1813(32.8 \%)$ rhinitis. There are higher chances of manifesting bronchial asthma and eczema, and rhinitis or all the three, if a student suffered from any one of these diseases. Students travelling to school by bus or van reported an increased risk of both eczema and rhinitis. Although the studies proves that the patient's belief of allergy on specific food item is wrong, in our study the students who reported food allergy were prone to get eczema. Students frequently exposed to

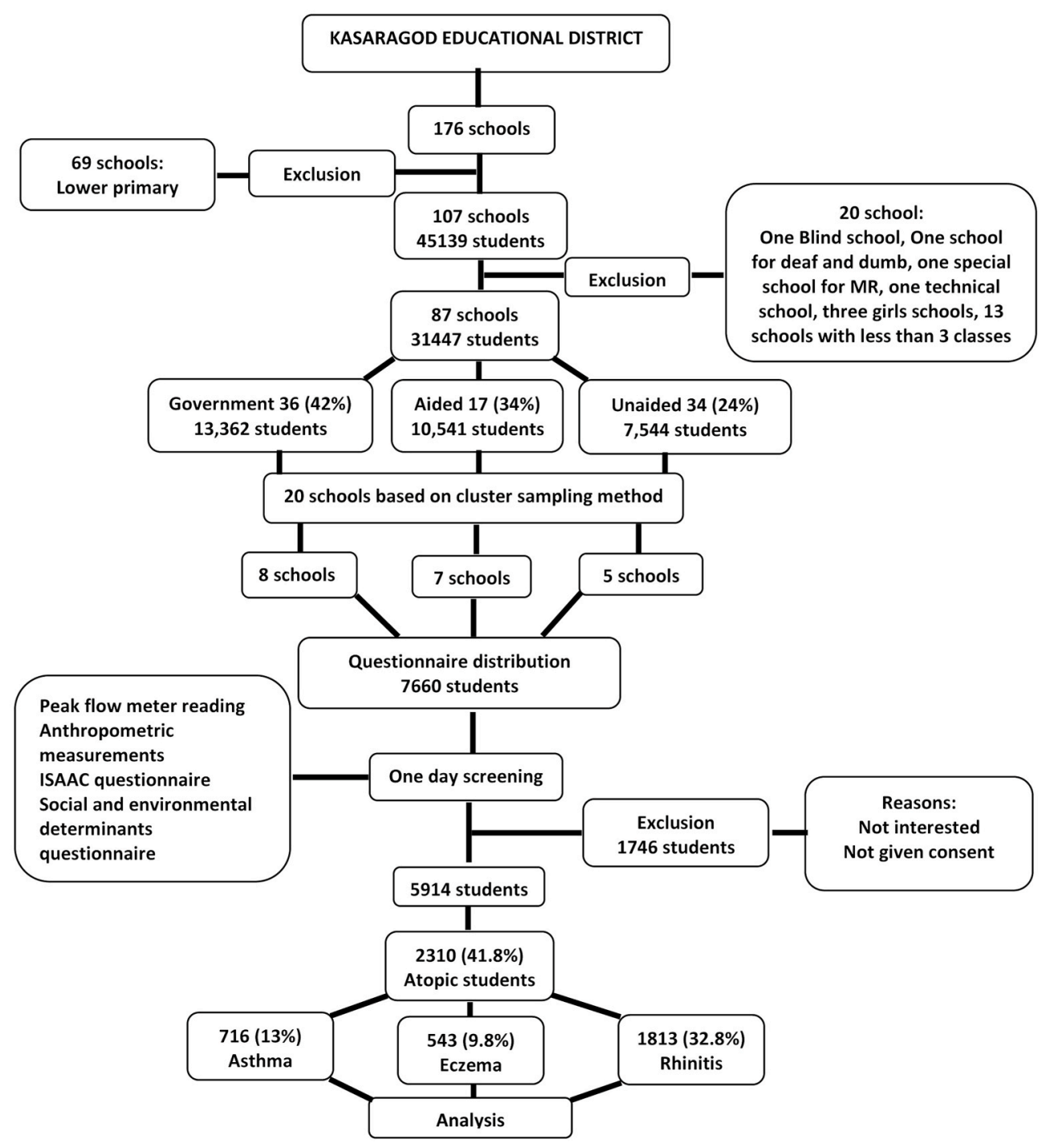

Fig. 1. Flow chart showing the student selection for the atopy prevalence study. 
Table 1

The environmental determinants of students with atopic disorders.

\begin{tabular}{|c|c|c|c|c|c|c|c|c|c|c|c|c|c|c|c|c|}
\hline \multicolumn{2}{|c|}{ Social and environmental determinants } & \multicolumn{5}{|l|}{ Asthma } & \multicolumn{5}{|l|}{ Eczema } & \multicolumn{5}{|c|}{ Rhinitis } \\
\hline & & $\mathrm{N}(\%)$ & $\mathrm{X}^{2}$ & $\mathrm{p}$ & OR & $95 \% \mathrm{CI}$ & $\mathrm{N}(\%)$ & $\mathrm{X}^{2}$ & $\mathrm{p}$ & OR & $95 \% \mathrm{CI}$ & $\mathrm{N}(\%)$ & $\mathrm{X}^{2}$ & $\mathrm{p}$ & OR & $95 \% \mathrm{CI}$ \\
\hline \multirow[t]{2}{*}{ Vehicular Traffic } & House near roads & $\begin{array}{l}603 \\
(84.2)\end{array}$ & 2.167 & 0.08 & 1.177 & $0.947-1.462$ & $\begin{array}{l}456 \\
(84)\end{array}$ & 2.043 & 0.088 & 1.194 & $0.936-1.522$ & $\begin{array}{l}1533 \\
(84.6)\end{array}$ & 4.367 & $0.021 * *$ & 1.184 & $1.010-1.388$ \\
\hline & $\begin{array}{l}\text { No roads (footpath, } \\
\text { waterways) }\end{array}$ & $\begin{array}{l}66 \\
(9.2)\end{array}$ & 1.492 & 0.126 & 0.843 & $.641-1.109$ & $\begin{array}{l}44 \\
(8.1)\end{array}$ & 0.002 & 0.51 & 0.993 & $0.718-1.374$ & $\begin{array}{l}164 \\
(9)\end{array}$ & 3.55 & $0.034^{* *}$ & 0.824 & $0.674-1.008$ \\
\hline \multirow[t]{6}{*}{$\begin{array}{l}\text { House to road } \\
\text { distance }\end{array}$} & Within $100 \mathrm{mts}$ & $\begin{array}{l}467 \\
(65.2)\end{array}$ & 1.668 & 0.796 & - & - & $\begin{array}{l}351 \\
(64.6)\end{array}$ & 8.356 & 0.079 & - & - & $\begin{array}{l}1212 \\
(66.9)\end{array}$ & 2.066 & 0.724 & - & - \\
\hline & Between 100 and & 125 & & & & & 114 & & & & & 296 & & & & \\
\hline & $500 \mathrm{~m}$ & (17.5) & & & & & (21) & & & & & (16.3) & & & & \\
\hline & Between 500 and & 16 & & & & & 12 & & & & & 38 & & & & \\
\hline & $1000 \mathrm{~m}$ & $(2.2)$ & & & & & $(2.2)$ & & & & & $(2.1)$ & & & & \\
\hline & More than $1000 \mathrm{~m}$ & $14(2)$ & & & & & $6(1.1)$ & & & & & $\begin{array}{l}29 \\
(1.6)\end{array}$ & & & & \\
\hline \multirow[t]{5}{*}{ House location } & Coastal region & $\begin{array}{l}75 \\
(10.5)\end{array}$ & 0.849 & 0.196 & 0.887 & $.686-1.145$ & $\begin{array}{l}52 \\
(9.6)\end{array}$ & 1.982 & 0.089 & 0.807 & $0.598-1.089$ & $\begin{array}{l}178 \\
(9.8)\end{array}$ & 7.322 & $0.004^{* *}$ & 0.776 & $0.646-0.933$ \\
\hline & Near the Paddy field & $\begin{array}{l}92 \\
(12.8)\end{array}$ & 6.985 & 0.137 & - & - & $\begin{array}{l}82 \\
(15.1)\end{array}$ & 0.764 & 0.943 & - & - & $\begin{array}{l}292 \\
(16.1)\end{array}$ & 4.655 & 0.325 & - & - \\
\hline & Near the Grain Mill & $\begin{array}{l}34 \\
(4.7)\end{array}$ & & & & & $\begin{array}{l}32 \\
(5.9)\end{array}$ & & & & & $\begin{array}{l}95 \\
(5.2)\end{array}$ & & & & \\
\hline & $\begin{array}{l}\text { Near Industrial } \\
\text { estate or } \\
\text { establishments }\end{array}$ & $\begin{array}{l}40 \\
(5.6)\end{array}$ & & & & & $\begin{array}{l}25 \\
(4.6)\end{array}$ & & & & & $\begin{array}{l}89 \\
(4.9)\end{array}$ & & & & \\
\hline & Near Cotton Mill & $\begin{array}{l}1 \\
(0.14)\end{array}$ & & & & & $3(0.6)$ & & & & & $\begin{array}{l}10 \\
(0.6)\end{array}$ & & & & \\
\hline $\begin{array}{l}\text { Exposure to } \\
\text { Vehicle/ } \\
\text { generator } \\
\text { smoke at home }\end{array}$ & $\begin{array}{l}\text { Diesel/petrol/ } \\
\text { kerosene }\end{array}$ & $\begin{array}{l}349 \\
(48.7)\end{array}$ & 1.525 & 0.116 & 0.906 & $.774-1.060$ & $\begin{array}{l}263 \\
(48.4)\end{array}$ & 0.819 & 0.195 & 0.921 & $0.772-1.100$ & $\begin{array}{l}804 \\
(44.3)\end{array}$ & 5.487 & $0.010^{*}$ & 1.144 & $1.022-1.281$ \\
\hline \multirow[t]{2}{*}{$\begin{array}{l}\text { Mode of going to } \\
\text { school }\end{array}$} & Bus/car/van/jeep & $\begin{array}{l}366 \\
(51.1)\end{array}$ & 1.009 & 0.167 & 0.923 & $.789-1.080$ & $\begin{array}{l}297 \\
(54.7)\end{array}$ & 6.844 & $0.005^{*}$ & 0.789 & $0.660-0.943$ & $\begin{array}{l}836 \\
(46.1)\end{array}$ & 11.439 & $0.000^{*}$ & 1.214 & $1.085-1.359$ \\
\hline & $\begin{array}{l}\text { Walking/auto/ } \\
\text { motor cycle/cycle }\end{array}$ & $\begin{array}{l}363 \\
(50.7)\end{array}$ & 0.344 & 0.292 & 1.048 & $.896-1.226$ & $\begin{array}{l}262 \\
(48.3)\end{array}$ & 2.904 & $0.049 * *$ & 1.167 & $0.977-1.393$ & $\begin{array}{l}983 \\
(54.2)\end{array}$ & 6.751 & $0.005^{*}$ & 0.861 & $.770-.964$ \\
\hline \multirow[t]{5}{*}{ House type } & Concrete & $\begin{array}{l}492 \\
(68.7)\end{array}$ & 3.489 & 0.625 & & & $\begin{array}{l}362 \\
(66.7)\end{array}$ & 6.033 & 0.303 & & & $\begin{array}{l}1224 \\
(67.5)\end{array}$ & 8.689 & 0.122 & - & - \\
\hline & Flats/Apartment & $\begin{array}{l}16 \\
(2.2)\end{array}$ & & & & & $\begin{array}{l}15 \\
(2.8)\end{array}$ & & & & & $\begin{array}{l}41 \\
(2.3)\end{array}$ & & & & \\
\hline & Sheet & $\begin{array}{l}6 \\
(0.84)\end{array}$ & & & & & $6(1.1)$ & & & & & $\begin{array}{l}17 \\
(0.93)\end{array}$ & & & & \\
\hline & Thatched & $5(0.7)$ & & & & & $6(1.1)$ & & & & & $\begin{array}{l}11 \\
(0.6)\end{array}$ & & & & \\
\hline & Tiled & $\begin{array}{l}175 \\
(24.4)\end{array}$ & & & & & $\begin{array}{l}127 \\
(23.4)\end{array}$ & & & & & $\begin{array}{l}461 \\
(25.4)\end{array}$ & & & & \\
\hline
\end{tabular}


Table 1 (continued)

\begin{tabular}{|c|c|c|c|c|c|c|c|c|c|c|c|c|c|c|c|c|}
\hline \multicolumn{2}{|c|}{ Social and environmental determinants } & \multicolumn{5}{|l|}{ Asthma } & \multicolumn{5}{|l|}{ Eczema } & \multicolumn{5}{|l|}{ Rhinitis } \\
\hline & & $\mathrm{N}(\%)$ & $\mathrm{X}^{2}$ & $\mathrm{p}$ & OR & $95 \% \mathrm{CI}$ & $\mathrm{N}(\%)$ & $\mathrm{x}^{2}$ & $\mathrm{p}$ & OR & $95 \% \mathrm{CI}$ & $\mathrm{N}(\%)$ & $\mathrm{x}^{2}$ & $\mathrm{p}$ & OR & $95 \% \mathrm{CI}$ \\
\hline \multirow[t]{2}{*}{ Ventilation } & Poor & $\begin{array}{l}39 \\
(5.4)\end{array}$ & 1.131 & 0.163 & 1.204 & $.855-1.697$ & $\begin{array}{l}42 \\
(7.7)\end{array}$ & 2.081 & 0.091 & 0.781 & $0.558-1.093$ & $\begin{array}{l}106 \\
(5.8)\end{array}$ & 1.079 & 0.164 & 1.133 & $0.895-1.433$ \\
\hline & Adequate & $\begin{array}{l}650 \\
(90.8)\end{array}$ & & & & & $\begin{array}{l}477 \\
(87.8)\end{array}$ & & & & & $\begin{array}{l}1634 \\
(90.1)\end{array}$ & & & & \\
\hline \multirow[t]{4}{*}{ Fuel } & Solid biomass fuels & $\begin{array}{l}318 \\
(44.4)\end{array}$ & 0.21 & $\begin{array}{l}0 . \\
.338\end{array}$ & 0.964 & $.823-1.129$ & $\begin{array}{l}230 \\
(42.4)\end{array}$ & 0.46 & 0.264 & 1.064 & $.889-1.273$ & $\begin{array}{l}878 \\
(48.4)\end{array}$ & 25.81 & $0.000^{*}$ & 0.746 & $0.666-0.835$ \\
\hline & $\begin{array}{l}\text { Domestic cooking } \\
\text { fuels and electricity }\end{array}$ & $\begin{array}{l}597 \\
(83.4)\end{array}$ & 0.009 & 0.48 & 1.01 & $.818-1.248$ & $\begin{array}{l}454 \\
(83.6)\end{array}$ & 0.005 & 0.5 & 0.991 & $0.780-1.259$ & $\begin{array}{l}1502 \\
(82.8)\end{array}$ & 0.844 & 0.189 & 1.073 & $0.923-1.247$ \\
\hline & Biogas & $\begin{array}{l}19 \\
(2.6)\end{array}$ & 0.364 & 0.311 & 0.859 & $.525-1.407$ & $\begin{array}{l}16 \\
(2.9)\end{array}$ & 0.984 & 0.196 & 0.765 & $0.450-1.301$ & $\begin{array}{l}51 \\
(2.8)\end{array}$ & 2.691 & 0.063 & 0.742 & $0.519-1.061$ \\
\hline & Kerosene & $\begin{array}{l}25 \\
(3.5)\end{array}$ & 0.006 & 0.936 & & & $\begin{array}{l}18 \\
(3.3)\end{array}$ & 0.029 & 0.865 & & & $\begin{array}{l}69 \\
(3.8)\end{array}$ & 1.083 & 0.168 & 0.852 & $0.631-1.152$ \\
\hline \multirow[t]{5}{*}{ Animal } & Domestic animals & $\begin{array}{l}88 \\
(12.3)\end{array}$ & 0.369 & 0.295 & 1.077 & $.848-1.366$ & $\begin{array}{l}80 \\
(14.7)\end{array}$ & 1.594 & 0.117 & 0.851 & $0.661-1.094$ & $\begin{array}{l}234 \\
(12.9)\end{array}$ & 0.022 & 0.459 & 1.013 & $0.857-1.197$ \\
\hline & Pets & $\begin{array}{l}297 \\
(41.5)\end{array}$ & 1.849 & 0.094 & 0.895 & $.763-1.050$ & $\begin{array}{l}224 \\
(41.3)\end{array}$ & 1.179 & 0.149 & 0.905 & $0.756-1.084$ & $\begin{array}{l}693 \\
(38.2)\end{array}$ & 1.007 & 0.165 & 1.061 & $0.945-1.191$ \\
\hline & Insects & $\begin{array}{l}608 \\
(84.9)\end{array}$ & 0.41 & 0.281 & 0.931 & $.748-1.159$ & $\begin{array}{l}451 \\
(83.1)\end{array}$ & 0.49 & 0.26 & 1.088 & $0.859-1.379$ & $\begin{array}{l}1500 \\
(82.7)\end{array}$ & 3.756 & $0.029 * *$ & 1.161 & $0.998-1.351$ \\
\hline & Rodents & $\begin{array}{l}489 \\
(68.3)\end{array}$ & 0.016 & 0.468 & 0.989 & $.836-1.171$ & $\begin{array}{l}377 \\
(69.4)\end{array}$ & 0.496 & 0.257 & 0.933 & $0.770-1.131$ & $\begin{array}{l}1289 \\
(71.1)\end{array}$ & 11.228 & $0.000^{*}$ & 0.811 & $0.718-0.917$ \\
\hline & Reptiles & $\begin{array}{l}637 \\
(89)\end{array}$ & 2.264 & 0.072 & 0.827 & $.645-1.060$ & $\begin{array}{l}472 \\
(86.9)\end{array}$ & 0.046 & 0.437 & 1.029 & $0.791-1.339$ & $\begin{array}{l}206 \\
(11.4)\end{array}$ & 4.901 & $0.014^{*}$ & 0.823 & $0.692-0.978$ \\
\hline $\begin{array}{l}\text { Allowing animals } \\
\text { inside the } \\
\text { home }\end{array}$ & & $\begin{array}{l}159 \\
(22.2)\end{array}$ & 0.227 & 0.333 & 1.048 & $.865-1.269$ & $\begin{array}{l}125 \\
(23)\end{array}$ & 0.823 & 0.197 & 1.104 & $0.892-1.366$ & $\begin{array}{l}387 \\
(21.3)\end{array}$ & 0 & 0.511 & 1 & $0.871-1.149$ \\
\hline $\begin{array}{l}\text { Allergy on } \\
\text { contact with } \\
\text { pets }\end{array}$ & & $\begin{array}{l}35 \\
(4.9)\end{array}$ & 2.499 & 0.073 & 1.349 & $.930-1.957$ & $\begin{array}{l}30 \\
(5.5)\end{array}$ & 4.617 & 0.025 & 1.542 & $1.036-2.294$ & $\begin{array}{l}60 \\
(3.3)\end{array}$ & 1.795 & 0.102 & 0.812 & $0.599-1.102$ \\
\hline \multirow[t]{2}{*}{ House dust mite } & $\begin{array}{l}\text { Not regularly } \\
\text { cleaning the bed, } \\
\text { pillow cover }\end{array}$ & $\begin{array}{l}72 \\
(10.1)\end{array}$ & 0.371 & 0.291 & 1.085 & $.835-1.410$ & $\begin{array}{l}60 \\
(11)\end{array}$ & 1.838 & 0.102 & 1.217 & $0.916-1.619$ & $\begin{array}{l}173 \\
(9.5)\end{array}$ & 0.036 & 0.442 & 1.019 & $.841-1.234$ \\
\hline & $\begin{array}{l}\text { Not regularly } \\
\text { washing cloths }\end{array}$ & $\begin{array}{l}53 \\
(7.4)\end{array}$ & 0.101 & 0.399 & 1.05 & $.777-1.418$ & $\begin{array}{l}37 \\
(6.8)\end{array}$ & 0.084 & 0.428 & 0.95 & $0.669-1.349$ & $\begin{array}{l}124 \\
(6.8)\end{array}$ & 0.314 & 0.308 & 0.939 & $0.753-1.171$ \\
\hline \multirow[t]{3}{*}{ Indoor pollution } & $\begin{array}{l}\text { Exposure to incense } \\
\text { sticks/dhoop }\end{array}$ & $\begin{array}{l}325 \\
(45.4)\end{array}$ & 0.037 & 0.44 & 1.016 & $.867-1.189$ & $\begin{array}{l}244 \\
(44.9)\end{array}$ & 0.152 & 0.366 & 1.036 & $0.867-1.238$ & $\begin{array}{l}846 \\
(46.7)\end{array}$ & 0.955 & 0.172 & 0.945 & $0.845-1.058$ \\
\hline & $\begin{array}{l}\text { Air fresheners/toilet } \\
\text { fresheners }\end{array}$ & $\begin{array}{l}163 \\
(22.8)\end{array}$ & 0.827 & 0.195 & 1.09 & $.905-1.314$ & $\begin{array}{l}121 \\
(22.3)\end{array}$ & 1.112 & 0.158 & 1.121 & $0.907-1.386$ & $\begin{array}{l}421 \\
(23.2)\end{array}$ & 1.196 & 0.144 & 1.077 & $0.943-1.229$ \\
\hline & $\begin{array}{l}\text { Body spray/ } \\
\text { perfumes }\end{array}$ & $\begin{array}{l}413 \\
(57.7)\end{array}$ & 0.862 & 0.187 & 1.078 & $.920-1.264$ & $\begin{array}{l}321 \\
(59.1)\end{array}$ & 0.006 & 0.486 & 1.007 & $0.841-1.206$ & $\begin{array}{l}1054 \\
(58.1)\end{array}$ & 1.444 & 0.121 & 1.072 & $0.957-1.202$ \\
\hline $\begin{array}{l}\text { Exposure to } \\
\text { Tobacco Smoke }\end{array}$ & & $\begin{array}{l}141 \\
(19.7)\end{array}$ & 0.353 & 0.292 & 1.062 & $.871-1.295$ & $\begin{array}{l}113 \\
(20.8)\end{array}$ & 1.391 & 0.132 & 1.141 & $0.916-1.421$ & $\begin{array}{l}353 \\
(19.5)\end{array}$ & 0.705 & 0.211 & 1.063 & $0.921-1.227$ \\
\hline
\end{tabular}

*Highly significant, ** Significant, $\mathrm{N}=$ number of students, $\mathrm{X}^{2}=$ chi square value, $\mathrm{OR}=$ odds ratio, $\mathrm{CI}=$ confidence interval. 
second-hand smoke from vehicle/generator at home, solid biomass fuels, rodents, reptiles had more prevalence of rhinitis. Rhinitis was significantly more common among students who lived nearer to the sea or tarred roads or footpath.

The prevalence of $\mathrm{AD}$ in children identified in 56 countries varies significantly from $0.3 \%$ to $20.5 \%$ but shows a consistent trend of increasing disease prevalence over time. ${ }^{1}$ We also determined PEFR in those students indicating the presence of bronchial asthma in the questionnaire. Out of 716 asthmatic students, 375 (52.5\%) students had clinically manifested asthma because of their low PEFR value. It is possible that other asthmatic students could be in remission during our visit. We did not make repeated visits to follow up other $47.5 \%$ students. Analysis of atopic students revealed the possibility of occurrence of asthma is also high in those of rhinitis students. There is a significant association between asthma and those who have a history of rhinitis or eczema and vice-versa; indicating atopic march among them, a need for focussed health education of parents for the long-term care of these children.

Multiple SEDs and psychological factors can trigger atopic disorders. They include environmental allergens and food hypersensitivity. The lower economic status of the family and poorer environmental conditions $^{9}$ has a maximum risk of atopy. The low economic status of the family showed a higher prevalence of allergic rhinitis in our students. Among atopic one form of the disease predisposed to the other in our students. Sehgal et al. showed that the temperature, humidity, food habits, clothing and psychological impacts on the clinical expression and severity of the disease. ${ }^{10}$ Our study showed no association with SEDs with bronchial asthma, and $47.5 \%$ of students showed a normal PEFR value indicating that more studies are needed to identify actual triggers. In asthmatic students, there is a significant relationship between PEFR and body weight, whereas no significant relation between height and PEFR. The finding shows that PEFR values are directly related to body weight, and obesity or malnutrition may be one of the possible aggravating factors for asthma. Generally, the PEFR is proportional to the height and gender of the students. ${ }^{11}$

A second-hand smoke exposure due to overcrowding in transport vehicles might be the cause of more prevalence of eczema \& rhinitis in students travelling to the school by van, bus or autorickshaw. Gawande and Kaware reviewed the consequences of fuel adulteration in India. They cited mixing Kerosene in petrol as the cause of air-borne diseases. ${ }^{12}$ As reported in earlier studies, the environmental factors for the onset of

Table 2

Logistic regression analysis of socio-environmental determinants found significant for rhinitis.

\begin{tabular}{|c|c|c|c|c|}
\hline $\begin{array}{l}\text { Socio-environmental } \\
\text { determinants }\end{array}$ & Wald & $\begin{array}{l}\mathrm{P} \\
\text { value }\end{array}$ & $\begin{array}{l}\text { Adjusted } \\
\text { OR }\end{array}$ & $95 \% \mathrm{CI}$ \\
\hline $\begin{array}{l}\text { Traffic: house near national and } \\
\text { state highways, district and } \\
\text { rural tarred roads }\end{array}$ & 2.496 & 0.114 & 1.209 & $0.955-1.530$ \\
\hline $\begin{array}{l}\text { Traffic: house near foot path, } \\
\text { waterways }\end{array}$ & 0.175 & 0.676 & 0.943 & $0.715-1.243$ \\
\hline Solid biomass fuels & 11.314 & $0.001^{\mathrm{a}}$ & 0.811 & $0.718-0.916$ \\
\hline $\begin{array}{l}\text { Mode of travel to school: bus, } \\
\text { van, car, jeep }\end{array}$ & 1.502 & 0.220 & 1.158 & $0.916-1.463$ \\
\hline $\begin{array}{l}\text { Mode of travel to school: } \\
\text { walking, auto, motorcycle }\end{array}$ & 0.370 & 0.543 & 1.076 & $0.850-1.361$ \\
\hline Insects & 2.986 & 0.084 & 1.160 & $0.980-1.372$ \\
\hline Rodents & 6.512 & $0.011^{\mathrm{a}}$ & 0.841 & $0.737-0.961$ \\
\hline Reptile & 5.142 & $0.023^{\mathrm{a}}$ & 0.798 & $0.657-0.970$ \\
\hline $\begin{array}{l}\text { Home situated in coastal } \\
\text { location }\end{array}$ & 6.191 & $0.013^{\mathrm{a}}$ & 0.790 & $0.656-0.951$ \\
\hline Type of school & 9.464 & $0.002^{\mathrm{a}}$ & 1.149 & $1.052-1.255$ \\
\hline Father's occupation & 0.096 & 0.757 & 1.002 & $0.988-1.017$ \\
\hline $\begin{array}{l}\text { Use of diesel, petrol, kerosene } \\
\text { vehicles/generator at home }\end{array}$ & 0.466 & 0.495 & 1.043 & $0.925-1.175$ \\
\hline Constant & 10.821 & 0.001 & 2.502 & \\
\hline
\end{tabular}

a Significant, $\mathrm{OR}=$ odds ratio, $\mathrm{CI}=$ confidence interval.
Table 3

Demographic details of the students participated in the survey.

\begin{tabular}{|c|c|c|}
\hline Demographic details & Categories & $\begin{array}{l}\text { No of students } \\
\text { (Percentage) }\end{array}$ \\
\hline \multirow[t]{2}{*}{ Gender } & Male & $2733(46.2)$ \\
\hline & Female & $3181(53.8)$ \\
\hline \multirow{3}{*}{$\begin{array}{l}\text { Annual family income } \\
\text { reported in the } \\
\text { questionnaire }\end{array}$} & Below INR 150000 & $5587(94.5)$ \\
\hline & $\begin{array}{l}\text { In between INR } 150001 \text { - } \\
350,000\end{array}$ & $121(2)$ \\
\hline & Above INR 350000 & $206(3.5)$ \\
\hline \multirow{2}{*}{$\begin{array}{l}\text { Financial status on the basis } \\
\text { of Kerala Government's } \\
\text { ration cards }\end{array}$} & APL & $3246(60.7)$ \\
\hline & BPL & $2100(39.3)$ \\
\hline \multirow[t]{17}{*}{ Father's occupation } & $\begin{array}{l}\text { Arts, Entertainment, Publishing } \\
\text { and Broadcasting }\end{array}$ & $15(0.3)$ \\
\hline & $\begin{array}{l}\text { Building, Landscape and } \\
\text { Construction Design }\end{array}$ & $92(1.6)$ \\
\hline & Health Care and Medical Science & $43(0.7)$ \\
\hline & Information Technology & $5(0.1)$ \\
\hline & Late & $43(0.7)$ \\
\hline & $\begin{array}{l}\text { Legal and Financial Services, and } \\
\text { Real Estate }\end{array}$ & $88(1.5)$ \\
\hline & $\begin{array}{l}\text { Managerial, Sales, Marketing } \\
\text { and HR }\end{array}$ & $125(2.1)$ \\
\hline & Natural Sciences and & $4(0.1)$ \\
\hline & Environmental Management & \\
\hline & Nil & $597(10.1)$ \\
\hline & Not applicable & $5(0.1)$ \\
\hline & Not mentioned & $116(2.0)$ \\
\hline & Personal Services & $4261(72.0)$ \\
\hline & $\begin{array}{l}\text { Postsecondary Education and } \\
\text { Knowledge Creation }\end{array}$ & $17(0.3)$ \\
\hline & $\begin{array}{l}\text { Primary/Secondary and } \\
\text { Vocational Education, } \\
\text { Remediation and Social Services }\end{array}$ & $116(2.0)$ \\
\hline & $\begin{array}{l}\text { Public Safety and Domestic } \\
\text { Security }\end{array}$ & $272(4.6)$ \\
\hline & $\begin{array}{l}\text { Skilled Production Workers: } \\
\text { Technicians, Operators, Trades, } \\
\text { Installers and Repairers }\end{array}$ & $115(1.9)$ \\
\hline \multirow[t]{14}{*}{ Mother's occupation } & $\begin{array}{l}\text { Building, Landscape and } \\
\text { Construction Design }\end{array}$ & $11(0.2)$ \\
\hline & Health Care and Medical Science & $55(0.9)$ \\
\hline & Housewife & 4525 (76.5) \\
\hline & Information Technology & $1(0)$ \\
\hline & $\begin{array}{l}\text { Legal and Financial Services, and } \\
\text { Real Estate }\end{array}$ & $50(0.8)$ \\
\hline & $\begin{array}{l}\text { Managerial, Sales, Marketing } \\
\text { and HR }\end{array}$ & $38(0.6)$ \\
\hline & Natural Sciences and & $5(0.1)$ \\
\hline & Environmental Management & \\
\hline & Personal Services & $734(12.4)$ \\
\hline & Not mentioned & $110(1.8)$ \\
\hline & $\begin{array}{l}\text { Postsecondary Education and } \\
\text { Knowledge Creation }\end{array}$ & $11(0.2)$ \\
\hline & $\begin{array}{l}\text { Primary/Secondary and } \\
\text { Vocational Education, } \\
\text { Remediation and Social Services }\end{array}$ & $303(5.1)$ \\
\hline & $\begin{array}{l}\text { Public Safety and Domestic } \\
\text { Security }\end{array}$ & $53(0.9)$ \\
\hline & $\begin{array}{l}\text { Skilled Production Workers: } \\
\text { Technicians, Operators, Trades, } \\
\text { Installers and Repairers }\end{array}$ & $18(0.3)$ \\
\hline \multirow[t]{3}{*}{ Food habits } & Vegetarian & $325(5.5)$ \\
\hline & Non-vegetarian & $320(5.4)$ \\
\hline & Mixed & $5079(85.9)$ \\
\hline \multirow[t]{3}{*}{ Staple food (5793 students) } & Rice & $5675(98)$ \\
\hline & Wheat & $18(0.03)$ \\
\hline & Rice and wheat & $100(1.7)$ \\
\hline
\end{tabular}

${ }^{\text {a }}$ Ration cards of 5346 students verified.

asthma and other allergic disorders were mainly cooking with Kerosene, tobacco smoke, traffic pollution, contact with pets, mud floor. ${ }^{13,14}$ As per government guidelines deworming tablets were given once in a year to the age group 1-19 years in all schools.

The non-participation rate was reasonably high (23\%) in our study. 
The reasons are; a) non-responsiveness of both students and their parents thinking that they do not have atopic disorders, b) non-cooperation of teachers in few schools and c) absentees at our target group. The known SEDs of asthma did not show any statistically significant relation in students of Kasaragod educational district. Endosulfan is an organochloride insecticide, which was sprayed aerially for cashew, paddy and other plants. There were residues of endosulfan found in soil, fruits and vegetables and human blood among the habitats of the area. Kasaragod is among the worst affected districts due to endosulfan areal spray. Our SED list did not include aerial endosulfan spray because the Government stopped the aerial spray and study itself screened students residing in the sprayed geographical area. Although a few studies mentioned bronchial asthma caused by endosulfan ${ }^{15}$ epidemiological evidence did not exist till date. High prevalence of the atopic disorder in Kasaragod compared to other places in India ${ }^{5}$ indicate a possible weak association with aerial spray. While analysing the SEDs between one school from endosulfan free panchayath and other schools, we did not find any statistically significant differences in the status of the prevalence of atopic disorders. There is also the possibility of students are travelling from endosulfan affected areas to this school, hence larger studies required to assess the impact of endosulfan in atopic and allergic disorders. Epigenetic changes due to aerial spray is one possibility of change in the phenotype in these students not studied till date. Students of Shimla showed the negative association of SEDs and exposure to smoking with asthma and prevalence rate of $2.3 \%{ }^{16}$ This also may indicate the importance of environmental pollution existing for a longer time as an essential factor for precipitating atopic disorders than a short period of exposure. We could not assess the psychological factors of the students due to the large sample size and the classroom setting of the study contributing to lack of time and privacy required for such assessment. This study is questionnaire based, hence reliability is less. Although an educational session was conducted prior to questionnaire administration, the possibilities of misinterpretation of terminologies used in the questionnaire, i.e., any dry or itchy rash is being classed as 'Eczema' and any form of rhinitis can be classified as allergic rhinitis. The consultation by experts and confirmatory tests for those who have shown positive to atopic disorders found beneficial. However, consultation was not done due to the huge sample size, constraints in funding and difficulty to get sufficient time during school hours. Although we have taken best of three PEFR reading from each students, there is a possibility of poor efforts intentionally or unintentionally.

We did not add the questions related to active smoking among children as the negative answer may not always be reliable in Indian culture. Increased tendency of using tobacco products in 10-15 years age group ${ }^{17}$ may be another cause for the increased prevalence of bronchial asthma.

\section{Conclusion}

The findings our study showed $41.8 \%$ prevalence of atopic disorders; bronchial asthma, allergic rhinitis and eczema in children with the age group of 10-15 years in Kasaragod educational district. Atopic students had tendency moving from one to other forms of the disease. Students exposed to solid biomass fuels, rodents and reptiles in the living places, houses situated in coastal location suffered from rhinitis, whereas eczema and asthma students were not affected by the major SEDs listed in the literature. Further studies to identify reasons for the high prevalence of atopic disorders focussed predominantly on environmental pollution along with the comprehensive health education to students, teachers and parents is recommended.

\section{Funding}

This work was supported by the Department of Environment and Climate change, Government of Kerala [Ref no:DoECC/E2/RD-45/658/ $2015 \backslash]$.
Table 4

Student reported allergy to food items and its symptoms.

\begin{tabular}{|c|c|c|}
\hline $\begin{array}{l}\text { Symptoms observed } \\
\text { after taking food }\end{array}$ & $\mathrm{N}(\%)^{*}$ & $\begin{array}{l}\text { Food items that caused specific food } \\
\text { allergy**\# }\end{array}$ \\
\hline Abdominal pain & $\begin{array}{l}187 \\
(36.2)\end{array}$ & $\begin{array}{l}\text { Vegetarian foods: } \\
\text { Refrigerated chilled food/water, oily } \\
\text { food, dhal, pea, biriyani, ghee rice, } \\
\text { chicken, milk, idli, spicy food, lemon } \\
\text { juice, biscuits, chapathi, rice flakes, } \\
\text { pickle, maida products, potato, } \\
\text { pineapple, pre night's left over food, } \\
\text { green gram. } \\
\text { Non vegetarian foods: } \\
\text { Egg, pork, beef, mutton, prawn }\end{array}$ \\
\hline Headache & $\begin{array}{l}66 \\
(12.8)\end{array}$ & $\begin{array}{l}\text { Vegetarian foods: } \\
\text { Refrigerated food, ghee, oily food, beans, } \\
\text { pea, biriyani, ghee rice, milk, lemon } \\
\text { juice, chapathi, rice flakes, chocolate, } \\
\text { date, palm, watermelon, refrigerated } \\
\text { water, } \\
\text { Non vegetarian foods: } \\
\text { Pork, egg, meat, }\end{array}$ \\
\hline Skin rashes & $\begin{array}{l}68 \\
(13.2)\end{array}$ & $\begin{array}{l}\text { Vegetarian foods: } \\
\text { Rose apple, brinjal, pineapple } \\
\text { Non vegetarian foods: shellfish, beef, } \\
\text { chicken, mutton, egg, fish, meat, pork, } \\
\text { squid }\end{array}$ \\
\hline Vomiting & $93(18)$ & $\begin{array}{l}\text { Vegetarian foods: } \\
\text { Bakery items, tea, oily food, sweet items, } \\
\text { soya bean, pea, junk food, spicy food, } \\
\text { cream cake, ice cream, soft drinks, egg } \\
\text { Non vegetarian foods: } \\
\text { Red meat, crab, prawn, pork, shellfish, } \\
\text { fish }\end{array}$ \\
\hline Rhinitis & $\begin{array}{l}44 \\
(8.5)\end{array}$ & $\begin{array}{l}\text { Vegetarian foods: } \\
\text { Bakery items, refrigerated food and } \\
\text { water, ghee, oil, citrus fruits, oily food, } \\
\text { milk, spicy food, fruit juices, chocolate, } \\
\text { ice cream } \\
\text { Non vegetarian foods: beef, chicken, } \\
\text { mutton, egg, fish }\end{array}$ \\
\hline Breathlessness & $\begin{array}{l}24 \\
(4.6)\end{array}$ & $\begin{array}{l}\text { Vegetarian foods: } \\
\text { Refrigerated food, citrus fruits, oily food, } \\
\text { biriyani, ghee rice, milk, sour food items, } \\
\text { ice cream, cucumber } \\
\text { Non vegetarian foods: shell fish, egg, } \\
\text { fish, }\end{array}$ \\
\hline Itching & $20(4)$ & $\begin{array}{l}\text { Vegetarian foods: } \\
\text { Bakery, refrigerated food, ghee, oil, citrus } \\
\text { fruits, } \\
\text { Non vegetarian foods: beef, chicken, } \\
\text { mutton, brinjal, egg, meat }\end{array}$ \\
\hline Body pain & $1(0.2)$ & Beef \\
\hline Gastritis & $5(1)$ & Egg \\
\hline Lip swelling & $2(0.4)$ & Crab, prawn, shell fish \\
\hline Chest pain & $9(1.7)$ & Oily food, fast food, junk food, egg \\
\hline
\end{tabular}

*517 patients. **based on students report of food items. \# None statistically significant.

\section{Acknowledgements}

We thank Deputy Director of Education, Kasaragod for permitting the study. We acknowledge the support of the head and teachers of twenty selected schools for conducting the study.

\section{References}

1 Pawankar R, Canonica GW, Holgate ST, Lockey RF. Executive Summary. Wisconsin: World Allergy Organisation. White Book on Allergy 2011-2012; 2011.

2 Jindal SK, Aggarwal AN, Gupta D, et al. Indian study on epidemiology of asthma, respiratory symptoms and chronic bronchitis in adults (INSEARCH). Int $J$ Tubercul Lung Dis. 2012;16:1270-1277.

3 Jain A, Vinod Bhat H, Acharya D. Prevalence of bronchial asthma in rural Indian children: a cross sectional study from South India. Indian J Pediatr. 2010;77:31-35.

4 Sharma BS, Kumar MG, Chandel R. Prevalence of asthma in urban school children in Jaipur, Rajasthan. Indian Pediatr. 2012;49:835-836. 
5 Narayana PP, Mitra PP, Narahari SR, Aggithaya MG. Prevalence of asthma in school children in rural India. Ann Thorac Med. 2010;5:118-119.

6 International Study of Asthma and Allergies in Childhood [homepage on the Internet]. Auckland, Global Asthma Network, University of Auckland. [updated 2017 Apr 17; cited 2020 June 23] Available from: http://isaac.auckland.ac.nz/index.html.

7 Chest research foundation [homepage on the Internet]. India: [updated 2018 May 16; cited 2018 Jul 9]. Available from: https://www.crfindia.com/.

8 Mathur R, ed. National Ethical Guidelines for Biomedical and Health Research Involving Human Participants. India: Indian Council of Medical Research; 2017.

9 Pakhale S, Wooldrage K, Manfreda J, Anthonisen N. Prevalence of asthma symptoms in 7th- and 8th-grade school children in a rural region in India. J Asthma. 2008;45: $117-122$.

10 Sehgal VN, Srivastava G, Aggarwal AK, Saxena D, Chatterjee K, Khurana A. Atopic dermatitis: a cross sectional (descriptive) study of 100 cases. Indian J Dermatol. 2015; 60:519.

11 Balasubramanian S, Ravikumar NR, Chakkarapani K, Shivbalan SO. Peak expiratory flow rate in children - a ready reckoner. Indian Pediatr. 2002;39:104-106.
12 Gawande AP, Kaware JP. Fuel adulteration consequences in India: a review. Sci. Revs. Chem. Commun. 2013;3(3):161-171.

13 Singh S, Sharma BB, Sharma SK, Sabir M, Singh V. ISAAC collaborating investigators. Prevalence and severity of asthma among Indian school children aged between 6 and 14 years: associations with parental smoking and traffic pollution. J Asthma. 2016; 53:238-244.

14 Vedanthan PK, Mahesh PA, Vedanthan R, Holla AD, Liu AH. Effect of animal contact and microbial exposures on the prevalence of atopy and asthma in urban vs rural children in India. Ann Allergy Asthma Immunol. 2006;96:571-578.

15 Anand AC. Kerala pesticide tragedy. Natl Med J India. 2001;14(2):123-124.

16 Behl RK, Kashyap S, Sarkar M. Prevalence of bronchial asthma in school children of 6-13 years of age in Shimla city. Indian J Chest Dis Allied Sci. 2010;52:145-148.

17 Tiwari RV, Megalamanegowdru J, Gupta A, et al. Knowledge, attitude and practice of tobacco use and its impact on oral health status of 12 and 15 year-old school children of Chhattisgarh, India. Asian Pac J Cancer Prev APJCP. 2014;15:10129-10135. 